SUPPORTING INFORMATION

\title{
The Great Reduction of Carbon Nanotube's Mechanical Performance by a Few Topological Defects
}

\author{
Liyan Zhu, Jinlan Wang, and Feng Ding
}

Table S1. Failure stress of perfect CNTs and those with a number of pentagon-heptagon $\mathrm{N}_{57}$ incorporated, Turning angle $\boldsymbol{\theta}\left({ }^{\circ}\right)$, Diameter $\boldsymbol{D}(\AA)$, Failure Stress $\boldsymbol{\sigma}(\mathrm{GPa})$, and equivalent breaking strain $\varepsilon$ and toughness $\mathbf{T}\left(10^{9} \mathrm{~J} / \mathrm{m}^{3}\right)$ to those of perfect CNTs.

\begin{tabular}{|c|c|c|c|c|c|c|}
\hline System & $\mathbf{N}_{57}$ & $\theta\left({ }^{\circ}\right)$ & D ( $(\AA)$ & $\sigma(\mathrm{GPa})$ & $\varepsilon_{\mathrm{e}}(\%)$ & $T_{e}\left(10^{9} \mathrm{~J} / \mathrm{m}^{3}\right)$ \\
\hline$(7,7)$ & 0 & 0.00 & 9.60 & 105.88 & 23.48 & 18.28 \\
\hline$(8,0)$ & 0 & 0.00 & 6.39 & 101.82 & 27.26 & 19.52 \\
\hline$(5,0)-(6,0)$ & 1 & 0.00 & 4.12 & 91.02 & 11.56 & 6.18 \\
\hline$(6,0)-(7,0)$ & 1 & 0.00 & 4.64 & 94.52 & 12.32 & 6.89 \\
\hline$(7,0)-(8,0)$ & 1 & 0.00 & 5.67 & 91.89 & 12.06 & 6.66 \\
\hline$(8,0)-(9,0)$ & 1 & 0.00 & 6.36 & 91.64 & 12.08 & 6.67 \\
\hline$(9,0)-(10,0)$ & 1 & 0.00 & 7.20 & 94.11 & 12.14 & 6.73 \\
\hline$(10,0)-(11,0)$ & 1 & 0.00 & 8.48 & 88.92 & 12.01 & 6.61 \\
\hline$(11,0)-(12,0)$ & 1 & 0.00 & 9.20 & 89.62 & 11.87 & 6.48 \\
\hline$(12,0)-(13,0)$ & 1 & 0.00 & 9.84 & 92.53 & 11.98 & 6.57 \\
\hline$(5,5)-(6,4)$ & 1 & 12.57 & 6.89 & 75.02 & 8.09 & 3.29 \\
\hline$(5,5)-(6,3)$ & 1 & 20.11 & 6.26 & 66.56 & 6.94 & 2.47 \\
\hline$(8,0)-(6,3)$ & 1 & 30.53 & 6.26 & 49.21 & 4.83 & 1.24 \\
\hline$(3,3)-(5,0)$ & 1 & 34.78 & 4.11 & 59.50 & 6.04 & 1.90 \\
\hline$(4,4)-(7,0)$ & 1 & 35.41 & 5.55 & 53.88 & 5.37 & 1.52 \\
\hline$(5,5)-(9,0)$ & 1 & 35.96 & 6.93 & 47.58 & 4.65 & 1.16 \\
\hline$(6,6)-(11,0)$ & 1 & 35.81 & 8.37 & 44.20 & 4.28 & 0.99 \\
\hline$(7,7)-(13,0)$ & 1 & 35.81 & 9.70 & 41.39 & 3.97 & 0.85 \\
\hline$(3,3)-(3,3)$ & 2 & 37.14 & 4.28 & 53.11 & 5.28 & 1.47 \\
\hline$(4,4)-(4,4)$ & 2 & 51.93 & 5.61 & 46.86 & 4.57 & 1.12 \\
\hline$(5,5)-(5,5)$ & 2 & 58.74 & 6.93 & 39.56 & 3.78 & 0.78 \\
\hline$(6,6)-(6,6)$ & 2 & 62.11 & 8.16 & 38.01 & 3.61 & 0.71 \\
\hline$(7,7)-(7,7)$ & 2 & 63.81 & 9.54 & 33.03 & 3.10 & 0.53 \\
\hline
\end{tabular}




\begin{tabular}{lllllll}
\hline & & & & & \\
$(6,0)-(6,0)$ & 2 & 45.98 & 4.64 & 50.55 & 4.98 & 1.32 \\
$(7,0)-(7,0)$ & 2 & 46.68 & 5.67 & 47.44 & 4.63 & 1.15 \\
$(8,0)-(8,0)$ & 2 & 63.80 & 6.36 & 30.93 & 2.88 & 0.46 \\
$(9,0)-(9,0)$ & 2 & 66.57 & 7.20 & 26.10 & 2.40 & 0.32 \\
$(10,0)-(10,0)$ & 2 & 53.14 & 8.48 & 43.14 & 4.16 & 0.93 \\
$(11,0)-(11,0)$ & 2 & 55.50 & 9.20 & 39.24 & 3.74 & 0.76 \\
$(12,0)-(12,0)$ & 2 & 55.84 & 9.84 & 37.02 & 3.51 & 0.67 \\
$(13,0)-(13,0)$ & 2 & 63.38 & 10.26 & 32.25 & 3.02 & 0.50 \\
\hline$(3,3)-(5,0)$ & 3 & 77.60 & 4.11 & 36.68 & 3.47 & 0.66 \\
$(4,4)-(7,0)$ & 3 & 82.18 & 5.55 & 34.27 & 3.22 & 0.57 \\
$(5,5)-(9,0)$ & 3 & 85.07 & 6.93 & 25.18 & 2.31 & 0.30 \\
$(6,6)-(11,0)$ & 3 & 92.62 & 8.29 & 26.54 & 2.45 & 0.33 \\
$(7,7)-(13,0)$ & 3 & 86.50 & 9.70 & 31.61 & 2.95 & 0.48 \\
\hline$(5,5)-(5,5)$ & 4 & 78.43 & 6.93 & 29.33 & 2.72 & 0.41 \\
$(9,0)-(9,0)$ & 4 & 89.06 & 7.20 & 24.73 & 2.27 & 0.29 \\
\hline
\end{tabular}



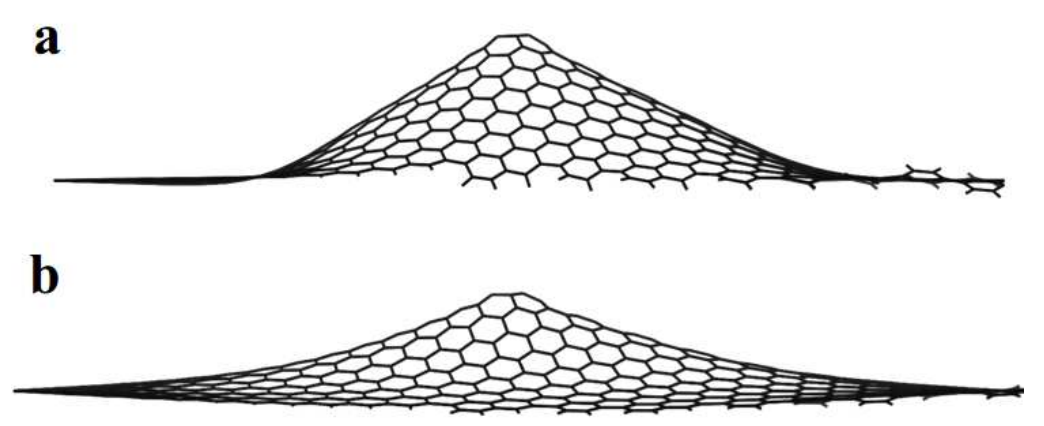

Figure S1. Snapshots of a graphene nanoribbon with an isolate pentagon under a strain of $15.0 \%$ (a) and $23.9 \%$ (b), respectively. 

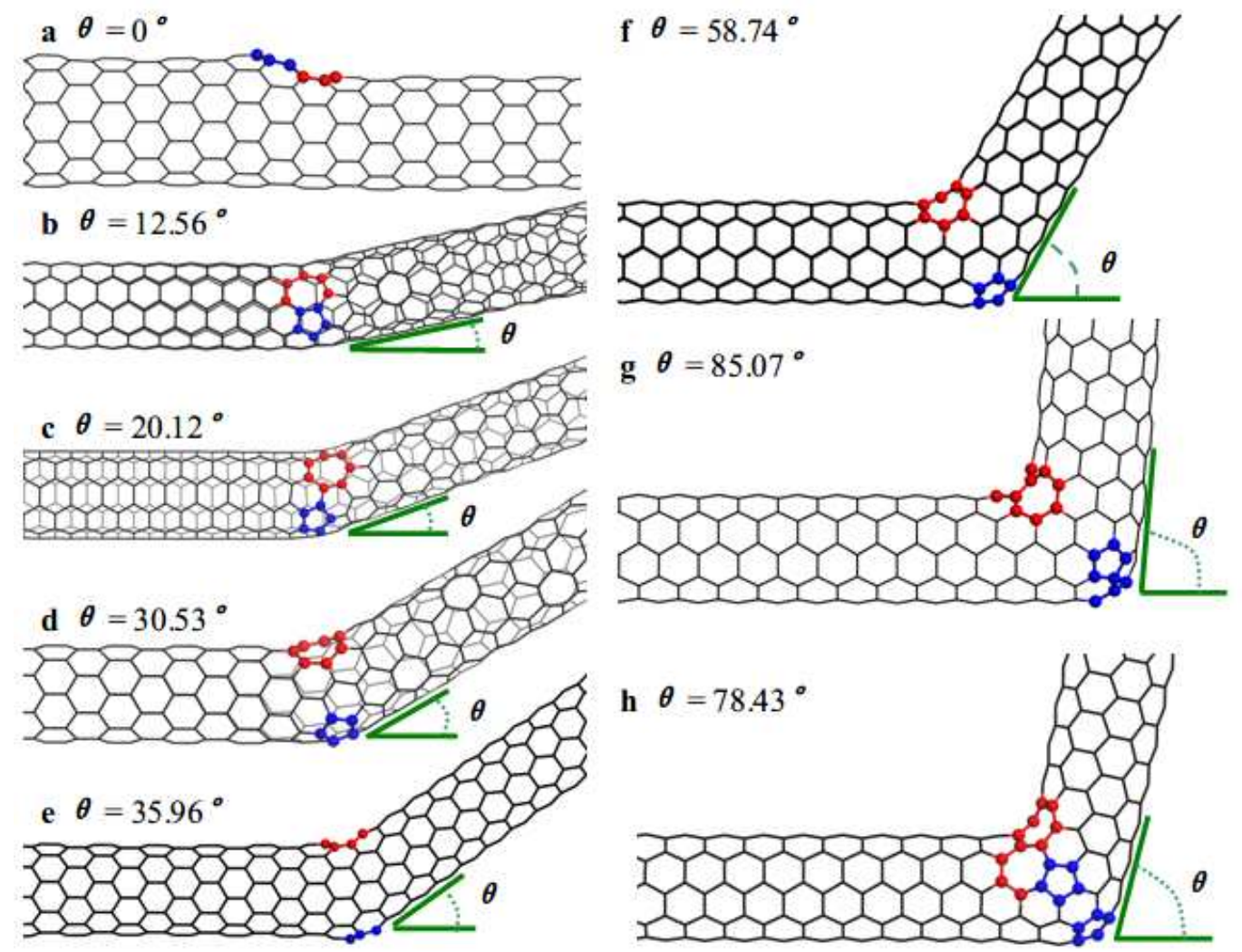

Figure S2. Geometries of CNTs with a few pairs of $5 \mid 7$ interposed. Turning angle $(\boldsymbol{\theta})$ is determined by the number of pairs of $5 \mid 7$ and the relative position between them. 


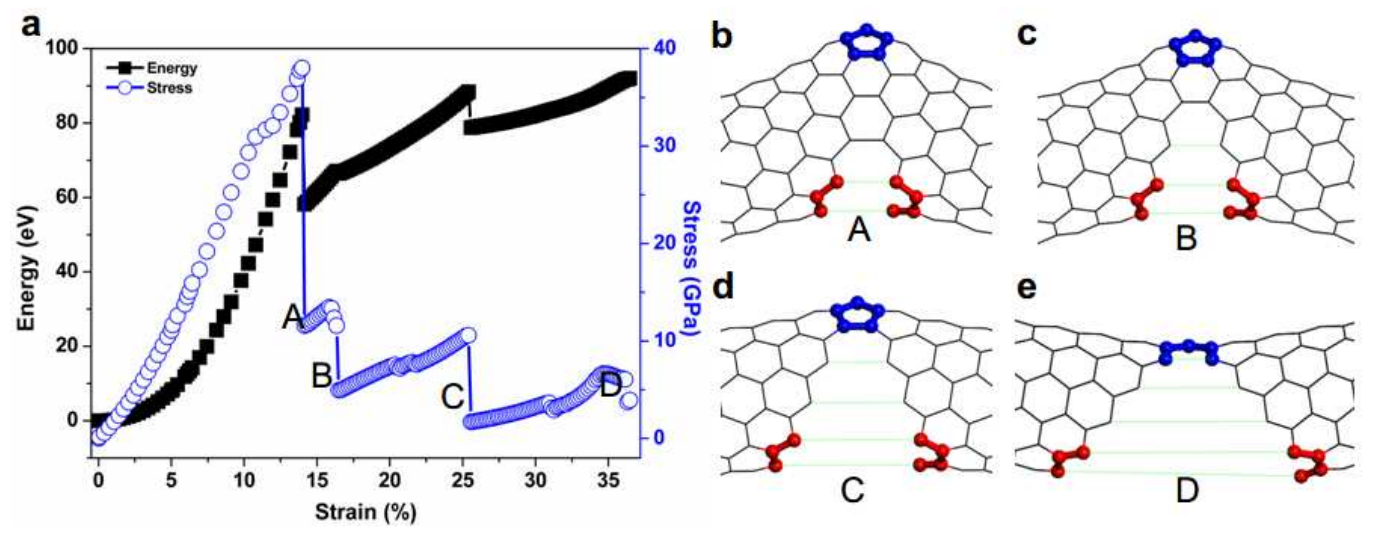

Figure S3. Strain energy and stress versus strain for $(6,6)-(6,6)-62 \cdot 11^{\circ}$ (a). Snapshots of elongated $(6,6)-(6,6)-62.11^{\circ}$ after the rupture of bonds (be).

5 

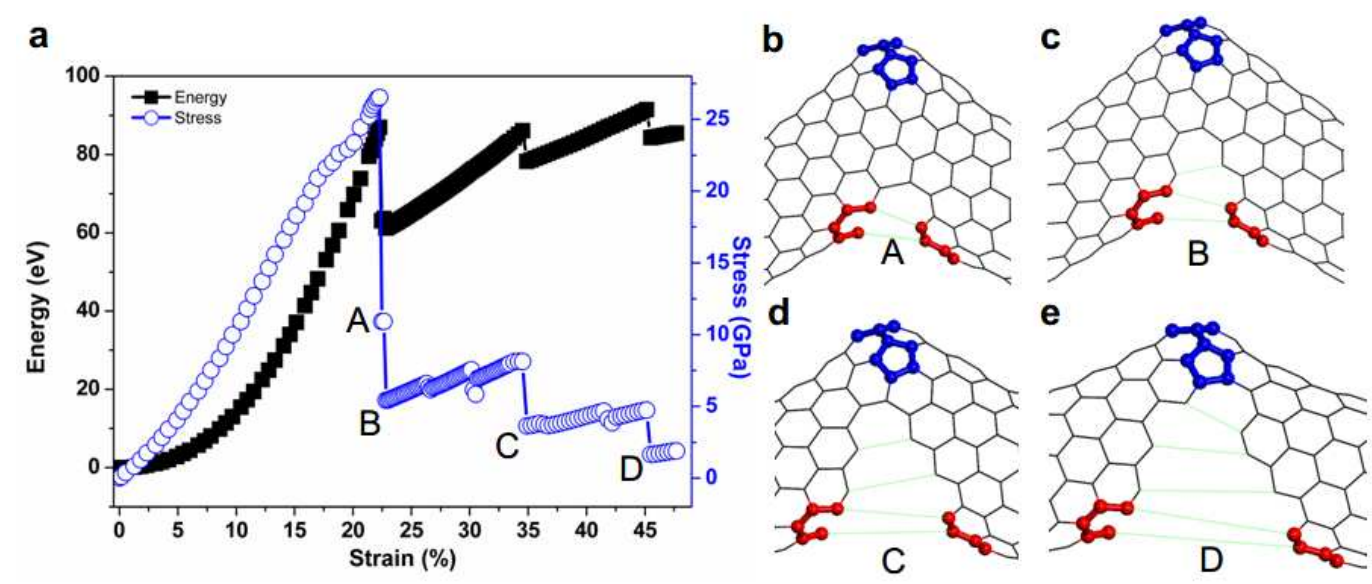

Figure S4. Strain energy and stress versus strain for $(6,6)-(11,0)-92.62^{\circ}$ (a). Snapshots of elongated $(6,6)-(11,0)-92.62^{\circ}$ after the rupture of bonds (b-e). 